\title{
Imatinib Preoperative Treatment for Gastrointestinal Stromal Tumors Clinical Analysis of 16 Cases
}

\author{
Zhao Xueyu, Li Dong hai and Liu Ming* \\ Department of General Surgery, Affiliated Hospital of Inner Mongolia Medical University, China

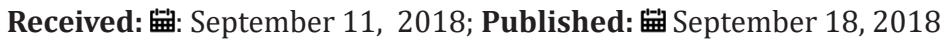 \\ *Corresponding author: Liu Ming, Department of General Surgery, Affiliated Hospital of Inner Mongolia Medical University, Huhhot, \\ P.R. China
}

\begin{abstract}
Objective To investigate the efficacy and safety of preoperative therapy with imatinib for unresectable/potentially resectable gaster and duodenum gastrointestinal stromal tumor (GIST). Methods The clinicopathological data of 16 patients diagnosed with unresectable/potential resectable gaster and duodenum GIST who received imatinib as preoperative therapy at the Affiliated Hospital of Inner Mongolia Medical University from July 2010 to June 2015 were retrospectively analyzed. Results The median time of preoperative therapy with imatinib was 7.5months. Image study for response assessment was achieved in all 16 cases, and all of them gained partial response PR after 3-9months preoperative therapy. Among them, 14 patients received R0 resection and 2 patients gave up surgery due to old age. During the preoperative treatment of imatinib, the incidence of adverse effect of grade 3 or above was $25.0 \%$ (4/16) , including 2 cases of neutropenia, 1 case of rash (grade 3), and 1 case of skin and digestive tract tissue edema (grade 4). The remaining patients were with grade 1 to 2 adverse effect. The median time of follow-up check was 47 (37-96) months, 3-year disease-free survival rate and overall survival rate are $81.25 \%$ and $100 \%$ respectively. Conclusion Preoperative imatinib therapy for unresectable/potentially resectable gaster and duodenum GISTs is generally well received. It is helpful to improve the surgical resection rate, reduce the operation range, avoid unnecessary joint organ resection, and protect the function of important structure. GIST is the most common mesenchymal tumor of the gastrointestinal tract [1].

The common onset sites are stomach, small intestine, colorectal, appendix, and esophagus. It may also occur in omentum, mesenteric, bladder, gallbladder, pancreas, retroperitoneal cavity, uterus, etc. [2]. Most GIST is caused by Cajal cell differentiation and have c-kit or PDGFRA receptor tyrosine kinase gene encoded activation mutations. In a few cases without c-kit or PDGFRA mutations, there are other molecular variants that may involve SDHX, BRAF, NF1, K/n-ras, and PIK3CA genes [1]. With the progress of molecular biological research, the treatment model has made a breakthrough. And the most important thing is the discover of the small molecule tyrosine kinase inhibitor imatinib, which can inhibit the growth of GIST cells by blocking the tyrosine kinase and promote apoptosis, thus to achieve good curative effect. But surgery is still an important means of treating GIST [3]. Since there was no obvious symptom in the early stage of GIST, about $30 \%$ of cases were unable to receive R0 resection when discovered [4]. As a result, preoperative imatinib treatment was used to make it possible to obtain a R0 resection of the GIST, and to improve the prognosis of patients. This study aims to investigate the efficacy and safety of preoperative therapy with imatinib for unresectable/potentially resectable gaster and duodenum GIST.
\end{abstract}

\section{Data and Methods}

\section{Inclusion and Exclusion Criteria}

A retrospective analysis of gastric and duodenal GIST patients from July 2010 to June 2015 at the Affiliated Hospital of Inner Mongolia Medical University was carried out, which was confirmed by biopsy or endoscopic guided biopsy. Preoperative treatment inclusion criteria

a) Surgical exploration or imaging evaluation of tumor involvement of important organs or vessels, and was estimated that it is difficult to achieve $\mathrm{R} 0$ resection

b) Massive tumor size $(>10 \mathrm{~cm})$, prone to bleeding and rupture in surgery and may cause iatrogenic dissemination c) The tumor is located in the gastroesophageal junction and duodenum, and surgery is likely to damage the function of important organs

d) The tumor can be removed but it is necessary to expand the range of excision, which may cause serious complications. Or high risk of recurrence and death after operation was expected

e) It is estimated that multi-organ resection should be performed. When one of the above conditions is satisfied and simultaneously satisfies the following conditions: the ECOG was less than 3, examination of blood and important organs (heart, lung, liver, kidney, bone marrow) have normal results; at least one measurable tumor not transformed to other organs; 
treatment of imatinib, or chemotherapy and radiotherapy, were not received before the group; good patient compliance, who are able to complete regular follow-up checks.

\section{Exclusion Criteria:}

a) If there is a genetic test, PDGFRA gene 18 exon D842V mutation

b) Second primary malignant tumors

c) Pregnant or lactation women

d) Suffering from other severe acute and chronic physiological or mental problems, which will increase the risk of participating in the study and use of drugs, or interfere with the results of the study.

\section{Included Object}

From July 2010 to June 2015, 236 cases of GIST were treated, of which 16 cases met the above conditions, 7 males and 9 females. The age ranged $37-81$ years and the median age is 57 years. ECOG score: remaining 5 cases were 3 points, 6 cases were 2 points, 5 cases were 1 point and 0 points. The primary site of the tumor: 11 cases were the stomach ( 2 of them were recurrence, 4 were located in the gastroesophageal junction), 5 were duodenal (1 of them was a recurrence). 13 of the cases were first diagnosis, 3 were postoperative recurrence with no distant metastasis. All 16 cases were classified as middle or high risk patients in preoperative evaluation. All patients were informed and had signed informed consent before treatment.

\section{Treatment Plan}

Enhanced CT or MRI was used as baseline before treatment. The patients took imatinib $400 \mathrm{mg}$ / d orally and were evaluated for curative effect regularly after taking the drug. When PR or when the maximum response is reached, timely assessment of whether R0 resection can be performed should be carried out. Patients who can undergo surgery will receive surgery after stopping the treatment for 1 - 2 weeks. Patients who cannot receive surgical treatment and SD would continue imatinib mesylate treatment. l weeks, 2 weeks, 1 month post treatment and every month afterwards, detailed symptoms, physical, ecog score, weight, blood routine and biochemical examination were evaluated. 3,6,9 months post treatment, patients receive enhanced CT examination to evaluate the curative effect. The CT scanning scope should include the whole abdominal cavity and pelvic cavity. The layer thickness is $5 \mathrm{~mm}$; Using axial images to measure the maximum diameter of the tumor. The whole CT value $\mathrm{Hu}$ ) of the tumor was obtained by curve edge tracing at the maximum level of the tumor during the enhancement venous phase.

\section{Evaluation Criteria}

a) The objective curative effect of the tumor was evaluated by choi standard [5]

b) CR: all measurable and unmeasured lesions disappeared without any new lesions. c) PR: CT examination shows that the tumor's long diameter decreases by $\geq 10 \%$, and / or the tumor's venous CT value $(\mathrm{Hu})$ decreases by $\geq 15 \%$; No new lesions were found. No obvious progress of the unmeasurable lesion was detected.

d) SD: does not conform to complete remission, partial remission or progress. And there was no worsening of symptoms caused by tumor progression.

e) PD: the long diameter of the tumor increased by more than or equal to $10 \%$, and the change of CT value in venous phase of the tumor did not meet the partial remission criteria; new lesions emerge; the size of new or existing intra-tumor nodules is increased. When the best overall curative effect is complete remission or partial remission, whether R0 surgery can be performed will be re-evaluated. The adverse effects were evaluated according to the American NCI - CTC classification standard version 4.0 and were classified into 1 - 5 grades.

\section{Statistics Processing}

Spss 19.0 statistical software was used for statistical analysis. Normal distribution measurement data were described by mean standard deviation $(\mathrm{X} \pm \mathrm{s})$, the deviation distribution measurement data were described by median. And the statistical analysis was performed by Kruskal-Wallis rank sum test. $\mathrm{P}<0.05$ was statistically significant.

\section{Result}

\section{Efficacy Assessment}

16 cases were evaluated 3, 6 and 9 months after treatment. 5 cases were SD (31.25\%) and 8 cases were PR (68.75\%) after 3 months of treatment. 1 case was SD (12.50\%) and 15 cases were PR (93.75\%) after 6 months of treatment. The drug continued to be administered to 5 cases up to 9 months, all of which achieved PR. In one typical case, after 6 months of medication, the tumor cells showed sparse density, extensive collagen and mucinous degeneration in the interstitium (Figures $1 \mathrm{a} \& 1 \mathrm{~b}$ ). The expression rate of cdll7 and dog-1 positive cells decreased significantly. As shown in figures, cdll7 decreased from $85 \%$ to $30 \%$ and dog-1 decreased from $80 \%$ to $23 \%$ (Figures 2 \& 3 ). The expression rate of Ki67 positive cells decreased from $17 \%$ to $4 \%$ (Figures $4 \mathrm{a} \& 4 \mathrm{~b}$ ), and the level of cell proliferation decreased significantly.

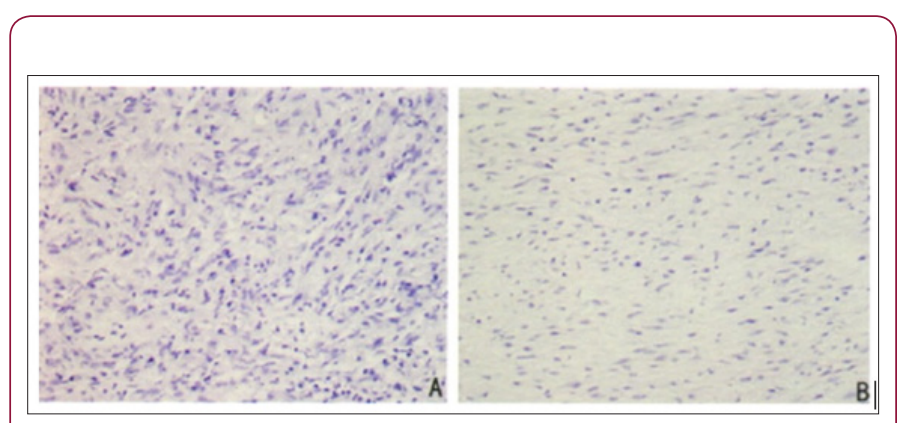

Figure 1: Histological changes before and after imatinib therapy (typical cases). 


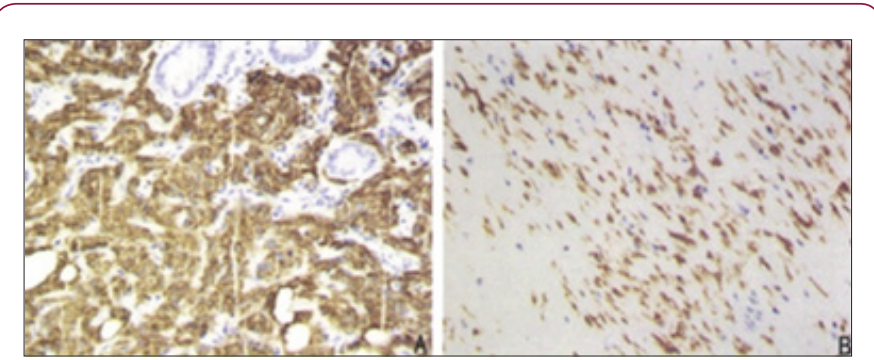

Figure 2: CD117 changes before and after treatment of imatinib (typical cases).

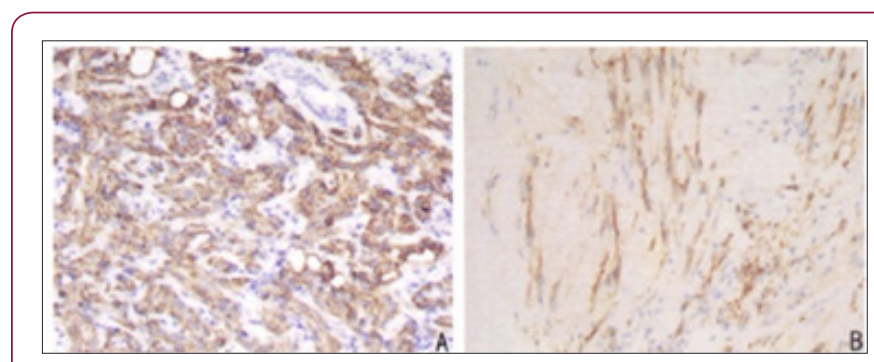

Figure 3: DOG-1 changes before and after treatment of imatinib (typical cases).

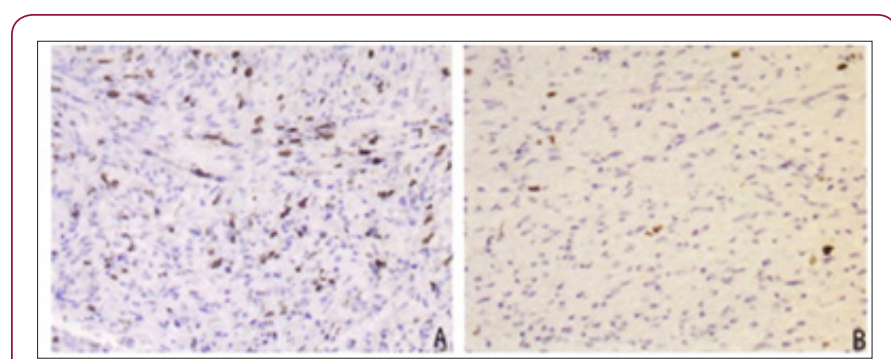

Figure 4: Ki-67 changes before and after treatment of imatinib (typical cases).

A is before treatment: fusiform cells are arranged in bundles, fences and vortices shapes, with abundant cells and visible mitotic images. (hematoxylin-eosin staining $\times 400$ ); B: after treatment: sparse cells, extensive collagen and mucinous degeneration in interstitium. (hematoxylin-eosin staining $\times 400$ ). A is $85 \%$ of CD117 positive cells before treatment (en vision staining $\times 400$ ); $\mathrm{B}$ is $30 \%$ cdll7 positive cells after treatment (en vision staining $\times 400$ ). A is $80 \%$ of dog- 1 positive cells before treatment (en vision staining $\times 400$ ); B is $23 \%$ dog-1 positive cells after treatment (en vision staining $\times 400$ ). A was $17 \%$ of Ki67 positive expression before treatment (en vision staining $\times 400$ ); B was $4 \%$ of Ki67 positive expression after treatment (en vision staining $\times 400$ ).

\section{Imaging Evaluation}

The diameter of the tumor was $95.7 \pm 34.5 \mathrm{~mm}$ and the CT value was $73.6 \pm 13.2 \mathrm{Hu}$ in all 16 patients before treatment. After 3, 6 and 9 months of treatment, the tumor diameter and CT value showed a decreasing trend. The difference of tumor size in each group was also statistically significant ( $\mathrm{x} 2=14.981, \mathrm{p}=0.001)$, and the difference of CT value in each group was statistically significant $(x 2=11.190$, $\mathrm{p}=0.004$ ). After comparing the two groups, the difference of tumor size and CT value were statistically significant $<0.05$. The tumor changes before and after treatment are shown in table 1 and (Figure 5). Among them, 2 cases had cystic changes after 3 months of treatment. Compared with 6 months of treatment, the maximum diameter and CT value of the tumors had no obvious changes, but were lower than before treatment. A for duodenal stromal tumor before treatment. B for the PR of duodenal stromal tumor 6 months after treatment (Table 1).

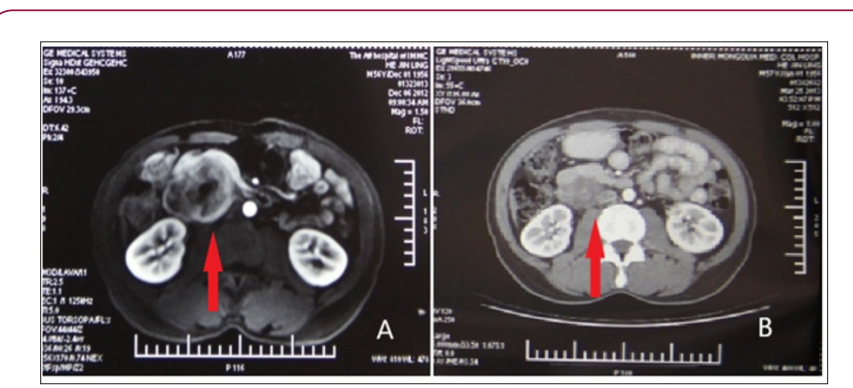

Figure 5: Imaging changes before and after treatment of imatinib.

Table 1: Diagnosis and treatment of gastrointestinal stromal tumors with imatinib mesylate in 16 cases of unresectable $\backslash /$ critical resection $(\mathrm{X} \pm \mathrm{s})$.

\begin{tabular}{|c|c|c|c|c|}
\hline Project & $\begin{array}{c}\text { Before } \\
\text { treatment }\end{array}$ & $\mathbf{3}$ months & $\mathbf{6}$ months & 9 months \\
\hline $\begin{array}{c}\text { Diameter } \\
(\mathrm{mm})\end{array}$ & $95.7 \pm 34.5$ & $77.9 \pm 29.6$ & $58.1 \pm 33.4$ & $42.2 \pm 27.3$ \\
\hline $\begin{array}{c}\text { CT value } \\
(\mathrm{Hu})\end{array}$ & $73.6 \pm 13.2$ & $58.4+12.4$ & $44.8+14.3$ & $39.8+12.5$ \\
\hline
\end{tabular}

\section{Operation Situation}

A total of 15 patients achieved PR after 6 months of preoperative treatment, 10 of whom were reevaluated as feasible for R0 surgical resection. However, due to age, 2 patients voluntarily gave up the operation. One of those was an 81-year-old male patient with gastric stromal tumors who maintained the progression of imatinib $400 \mathrm{mg} / \mathrm{d}$ for 12 months, increasing to $600 \mathrm{mg} / \mathrm{d}$. The other was a 76-year-old female patient with duodenal stromal tumors maintained the progress of imatinib $400 \mathrm{mg} / \mathrm{d}$ for 18 months, and switched to sunitinib $37.5 \mathrm{mg} / \mathrm{d}$.

The other 8 patients underwent R0 operation. The remaining 6 cases were re-evaluated as feasible for R0 surgery after 9 months of preoperative treatment. The median preoperative treatment time for the 14 patients was 7.5 months. Of the 14 patients who underwent surgical resection, 6 had a high risk of preoperative evaluation. It may be difficult to achieve complete $\mathrm{R} 0$ resection for those who underwent the surgery. 4 had to undergo partial resection for tumors that needed extensive surgery. 2 had retained the function of spleen and cardia at the esophagogastric junction. 2 high risk patients had survived the perioperative period safely.

\section{Adverse Effects}

Imatinib has a quick response, mild adverse effect and good tolerance. During the treatment period, the incidence of grade 3 or above adverse effect was $25.0 \%$ (4/16), including 2 cases of grade 3 leukopenia, 1 case of grade 3 rash, and 1 case of grade 4 skin and 
digestive tract edema (serious digestive tract and skin edema). One year after the operation, the edematous tissue at the closed end of the gastric wall was cut by stapler and gradually formed a fistula at the closed end of the stomach wall. Most of the other adverse effects were 1 - 2 degrees, which could be improved after symptomatic treatment. The main adverse effects were: 2 cases of thrombocytopenia; 1 case of hemoglobin decrease; Liver function damage in 3 cases; Renal function damage in 2 cases; 3 cases of diarrhea; Nausea and vomiting occurred in 5 cases. Asthenia in 7 cases; loss of appetite in 5 cases; Muscle soreness in 3 cases; 3 cases of rash; Edema of skin mucosa in 5 cases. None of the 16 cases showed intolerance to adverse effects to stop treatment.

\section{Follow-up examination status}

All 16 patients were followed up till June 2018, followed by a median time of 47 (37-96) months. There was tumor progression in 5 cases $(31.25 \%)$ and death in 2 cases $(12.5 \%)$. No disease survival rate and total survival rate were $81.25 \%$ and $100 \% 3$ years post operation. Among the 14 patients who underwent surgery, the median time of disease-free survival was 44 (6-96) months, and the total survival median time was 47 (37-96) months.

\section{Discussion}

The treatment before surgery can be called preoperative treatment. For a long time, there has been some confusion in the concepts of neoadjuvant therapy, induction therapy, initiation therapy, and conversion therapy. The concept of preoperative treatment should be decided by the timing of treatment. Therefore, the term " neoadjuvant treatment" is no longer used in nccn soft tissue sarcoma treatment guidelines, esmo stromal tumor treatment guidelines, and the 2017 edition of gist diagnosis and treatment with Chinese experts' consensus. Preoperative treatment was used instead [3]. The advantages of preoperative treatment include:

a. reducing tumor size and clinical staging.

b. Narrowing the scope of operation, avoiding unnecessary joint organ resection, reducing the risk of operation, and increasing the chance of radical resection.

c. The structure and function of important organs can be protected from the tumor in special position.

d. The possibility of iatrogenic dissemination can be reduced when the tumor is large, with greater risk of rupture and bleeding. At the same time, in the 2018 edition of the NCCN guide, mutation detection of the KIT and PDGFRA genes of the biopsies for pre-operative treatment, and the detection of the embryo line mutation of the SDH gene for the absence of the above two mutations (wild type) were strongly recommended. Whether or not to carry out preparation and the dosage of preoperative treatment should be determined [6].

The RTOG0132/ACRIN6665 experiment [7] is the first prospective study of GIST preoperative treatment. The cases in the multi center stage II trial group were medium-high risk and recurrent GIST. And imatinib (600 mg/d) is applied for 8-12 weeks before operation. The study shows that preoperative treatment can significantly increase the rate of R0 resection by $77 \%$. The results showed that the total survival rate was $77 \%$ in 5 years, and imatinib was well tolerated and safe to use before operation. APOLLON (NCT00112632) [8] is another multicenter prospective phase II experiment. The standard of the group is potentially resectable middle risk GIST patients. In the previous experiment data, 83\% of the $\mathrm{R} 0$ resection rate was obtained after an average of 9.4 months of preoperative treatment. Sumin Tang et al. Study [9] showed that 28 cases of GIST patients received preoperative treatment on average of 13.5 months before operation and 24 cases (85.7\%) had partial remission.

The time of tumor reduction was mainly 6-12 months. The average diameter before treatment was $10.5 \mathrm{~cm}$, and after preoperative treatment, it was reduced to $5.9 \mathrm{~cm}$. And the $\mathrm{R} 0$ resection rate was $94.7 \%$. At present, there are few reports on the treatment of gastric and duodenal stromal tumors. In this study, 16 cases of non resectable / potentially resectable gastric and duodenal stromal tumors were treated with imatinib. The results of the study were similar to those of other studies. The proportion of partial remission was high. After the preoperative treatment, 14 of the GIST patients received the operation. R0 excision was obtained in all GIST patients. However, due to the small number of cases, clinical experience should be continually accumulated. In terms of the safety of preoperative treatment, patients generally tolerated imatinib. And there were no significant adverse effects on the safety to surgery. Demetri and other reports showed that the incidence of severe toxicity ( 3 or 4 degrees) was $21.1 \%$. The most serious adverse effect occurred mainly in gastrointestinal bleeding or intraperitoneal bleeding among patients with larger tumor size, accounting for $5 \%$. And most of the other adverse effects were 1 or 2 degrees.

According to Yang Hong Xin and other [10,11] in 96 cases of advanced and high-risk GIST patients that received imatinib, the main toxic and side effects were edema (74.0\%), nausea (56.3\%), abdominal discomfort (53.1\%), leukocyte decline (44.8\%), anemia (29.2\%) and so on, mainly with 1-2 degree of toxicity. There were 8 patients with 3rd degree toxic reaction, which can be improved after symptomatic treatment. 4 cases had reduction of dosage to $300 \mathrm{mg} / \mathrm{d}$. And there was no toxic side effects or serious complications. This study showed that most of the patients had mild side effects and good tolerance. 1 patient received imatinib before and after operation had severe tissue edema. After one year, the edemations tissue of the closed end of the gastric wall was cut by stapler and gradually formed an abdominal fistula at the closed end of the stomach. One year later, the fistula was closed by surgery. Close observation and timely intervention of severe adverse effects are helpful to the implementation of preoperative adjuvant treatment and the safety of patients.

The accurate evaluation of preoperative treatment of GIST not only determines the timing of surgical treatment, but also objectively reflects the efficacy of preoperative drug treatment, 
providing a reference for adjuvant treatment. Preoperative evaluation was mainly based on enhanced CT/MRI. The evaluation criteria included RECIST, WHO, SWOG and Choi. In the case of GIST, the tissue composition of the target therapy is early and varied. It can be manifested as cystic change, mucus change, bleeding and necrosis. The size of tumor may not be reduced significantly, and the significant increase of liquid components caused by cystic change or mucus change even lead to a significant increase in tumor size, so RECIST standard [12] does not apply here. This standard introduces changes in CT values into the evaluation index. The choi standard stipulates that if the CT values drop by more than $15 \%$ after treatment, even if the size increases, the treatment should be considered effective, thus improving the accuracy and sensitivity [13]. It is recommended by the NCCN guide and the consensus of Chinese experts. In this study, the diameter and CT value of the tumor changed with preoperative treatment, and the difference was statistically significant. The Choi standard was more applicable than the other criteria for GIST.

Until now, there is still a lot of controversy about the timings of preoperative treatment, operation opportunity, and postoperative medication. The main reason is the variety of GIST gene mutation, and different sensitivity to imatinib. Meanwhile, drug resistance will occur during the treatment. Studies have shown that a significant reduction of GIST's size often occurs within 4-6 months after taking the drug. After taking the medicine for 9 months, the tumor size continued to decrease inconspicuously, and the maximum response to treatment may need 6 to 12 months [14]. Chen Si-le [15] showed that the average time of preoperative treatment was 6 (1-18) months. Recently [16], in a Japan and South Korea multicenter study, a high rate of R0 resection and low toxicity for huge gastric GIST ( $>10 \mathrm{~cm}$ ) after 6-9 months of preoperative imatinib treatment was reported. The median time of preoperative treatment was 7.5 months, which was similar to that of the above study. Therefore, for preoperative treatment period, NCCN guidelines recommend " 6 months or maximum response time"; ESMO guidelines recommend "6 to 12 months or maximum response time".

The recommendation of the Chinese expert consensus was changed from the " 6 months" in the 2013 edition to the "6-12 months" in the 2017 edition. However, the risk of drug resistance may occur and the best timing for treatment is lost because of too long preoperative treatment. Therefore, it is not recommended that the preoperative treatment should be longer than 1 year. The other important question is how to determine the period for postoperative adjuvant treatment in patients with preoperative treatment. Research on such questions is very limited and further study is still needed. To sum up, preoperative treatment has been recognized as one of the standard models of clinical treatment of unresectable/potential resectable GIST. For the unresectable/ potential resectable gastric and duodenal GIST preoperative treatment, it is safe and effective, and can obtain effective tumor reduction, which helps to reduce the extent of the operation, retain the function of important organs, and reduce the complications of the operation. The incidence of serious adverse effects of imatinib is low, but it should be closely observed. If serious adverse effects occur, close attention should be paid to them.
However, there are still disputes about the length of preoperative treatment; timing of operation; whether the preoperative treatment can improve the overall survival; following the preoperative treatment, how to continue treatment after surgery; and the effectiveness of preoperative treatment for resectable patients whose initial assessment is moderate to high risk. The number of cases in this study is relatively small, and we hope to have a larger sample and more comprehensive study for further demonstration.

\section{References}

1. Li J, Ye Y, Wang J, Zhang B, Qin S, et al. (2017) Chinese consensus guidelines for diagnosis and management of gastrointestinal stromal tumor. Chinese Journal of Cancer Research 29(4): 281-293.

2. Soreide K, Sandvik OM, Soreide JA, Giljaca V, Bulusu VR, et al. (2016) Global epidemiology of gastrointestinal stromal tumours (GIST): A systematic review of population-based cohort studies. Cancer Epidemiology 40: 39-46.

3. LI Zi yu, LI Shuang xi, JI Jia fu (2018) Application of preoperative target therapy and the timing of surgery for gastrointestinal stromal tumor. Chinese Journal of Practical Surgery 38(05): 494-497.

4. Chen Chun hua, Liu Ming (2014) Imatinib mesylate preoperative treatment for gastrointestinal stromal tumor: clinical analysis of 12 cases. International Journal of Surgery 41(7): 460-463.

5. Choi H (2008) Response evaluation of gastrointestinal stromal tumors. Oncologist 13(Supp 2): 4.

6. Zhao Gang, Wang Ming (2018) Updates and interpretations of the NCCN Clinical Practice Guidelines (2018 first version) on gastrointestinal stromal tumor. Chinese Journal of Practical Surgery 38(5): 515-519.

7. Eisenberg BL, Harris J, Blanke CD, Heinrich MC, Watson JC, et al. (2009) Phase II trial of neoadjuvant/adjuvant imatinib mesylate (IM) for advanced primary and metastatic/recurrent operable gastrointestinal stromal tumor (GIST): early results of RTOG 0132/ACRIN 6665. Annals of Surgical Oncology 99(1): 42-47.

8. Hohenberger P, Langer C, Wendtner CM, Werner H, Annette P, et al. (2012) Neoadjuvant treatment of locally advanced GIST: Results of APOLLON, a prospective, open label phase II study in KIT- or PDGFRApositive tumors. J Clin Oncol 30 (suppl): 10031.

9. Tang S, Yin Y, Shen C, Chen J, Yin X, et al. (2017) Preoperative imatinib mesylate (IM) for huge gastrointestinal stromal tumors (GIST). World Journal of Surgical Oncology 15(1): 79.

10. Demetri G D, Von MM, Blanke CD, Annick D, Burton E, et al. (2002) Efficacy and safety of imatinib mesylate in advanced gastrointestinal stromal tumors. N Engl J Med 347(7): 472-480.

11. Yang Hong xin (2017) Clinical efficacy and safety of imatinib in treatment of advanced and high-risk gastrointestinal stromal tumor. Chinese Journal of general surgery 26(4): 437-442.

12. Tang Lei (2017) Radiological Evaluation of the Response of Abdominal Tumors to Targeted Therapy: Current Status and Prospect. Medical Journal of Peking Union Medical College Hospital 8(z1): 82-89.

13. Shin S, Choi H (2008) Imaging and Response Evaluation of Gastrointestinal Stromal Tumors. Oncologist 13 Suppl 2(Supplement 2): 4.

14. De Azevedo CR, Jr PT, Rossi BM, Guimaraes GC, De Souza B, et al. (2011) Pathologic complete response with neoadjuvant imatinib for locally advanced pelvic GIST. International Journal of Clinical Oncology 16(3): 279-283.

15. Chen Si le, Song Wu, Peng Jian Jun (2018) Analysis of the effectiveness of imatinib preoperative adjuvant therapy in 23 patients with locally advanced gastrointestinal stromal tumor. Chinese Journal of Practical Surgery 38(5): 546-550.

16. Kurokawa Y, Yang HK, Cho H, Ryu MH, Masuzawa T, et al. (2017) Phase II study of neoadjuvant imatinib in large gastrointestinal stromal tumours of the stomach. British Journal of Cancer 117(1): 25-32. 
ISSN: 2574-1241

DOI: 10.26717/BJSTR.2018.09.001756

Liu Ming. Biomed J Sci \& Tech Res

(C) (i) This work is licensed under Creative

Submission Link: https://biomedres.us/submit-manuscript.php

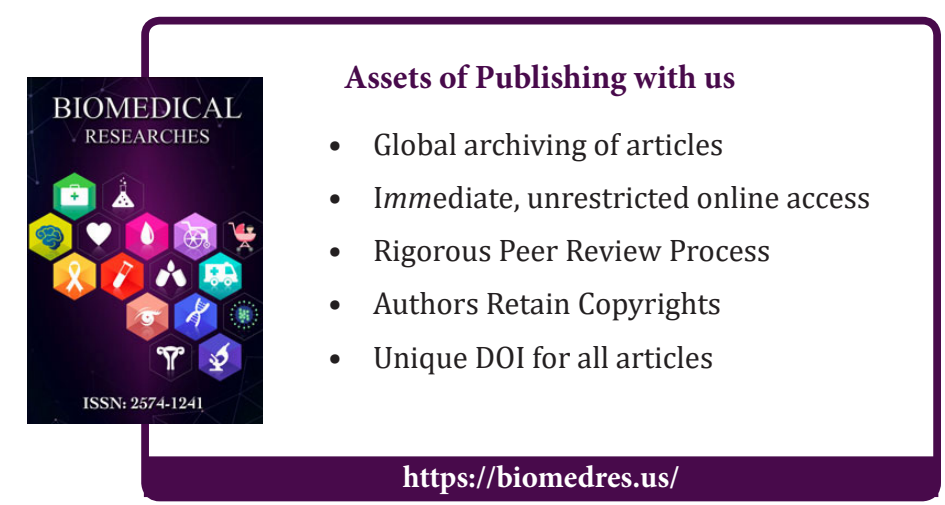

